\title{
A universal Dirac operator and noncommutative spin bundles over fuzzy complex projective spaces
}

\author{
Brian P. Dolan, ${ }^{1,2 *}$ Idrish Huet, ${ }^{1,3 \dagger}$ \\ Seán Murray ${ }^{1,2 \ddagger}$ and Denjoe O’Connor ${ }^{1 \S}$
}

1 School of Theoretical Physics, Dublin Institute for Advanced Studies, 10 Burlington Road, Dublin 4, Ireland.

2 Department of Mathematical Physics, NUI Maynooth, Maynooth, Co. Kildare, Ireland.

${ }^{3}$ Depto de Física, Centro de Investigación y de Estudios Avanzados del IPN, Apdo. Postal 14-740, 07000 México D.F., México.

November 8, 2007

\begin{abstract}
We present a universal Dirac operator for noncommutative spin and $\operatorname{spin}^{\mathrm{c}}$ bundles over fuzzy complex projective spaces. We give an explicit construction of these bundles, which are described in terms of finite dimensional matrices, calculate the spectrum and explicitly exhibit the Dirac eigenspinors. To our knowledge the $\operatorname{spin}^{\mathrm{c}}$ spectrum for $\mathbb{C P}^{n}$ with $n \geq 3$ is new.
\end{abstract}

*email: bdolan@thphys.nuim.ie

†email: mazapan@stp.dias.ie

†email: smury@stp.dias.ie

$\S$ email: denjoe@stp.dias.ie 


\section{Introduction}

There has been a growing interest in recent years in noncommutative geometry and its applications to physics, especially quantum field theory [1] and string theory $[2,3]$. This paper focuses on a particular kind of noncommutative geometry and associated spaces, known as fuzzy spaces, which serve as regulators in field theories [4]. The advantage of this approach over the traditional lattice discretisation lies in the fact that fuzzy spaces retain the isometries of the underlying space. Fuzzy spaces are finite approximations to suitable continuum manifolds: more precisely a fuzzy space can be defined by a sequence of finite dimensional algebras $\mathcal{A}_{L}$ that approximate the commutative algebra of functions on the manifold $\mathcal{M}$ as $L \rightarrow \infty$, together with some differential geometric information such as a Laplacian or a Dirac operator. The archetypical algebra $\mathcal{A}_{L}$ being the full matrix algebra of size $d_{L}, \operatorname{Mat}_{d_{L}}(\mathbb{C})$.

So far several fuzzy spaces have been constructed since the introduction of the seminal example, the fuzzy 2 -sphere $[5,6,7]$ and various field theories have been studied within this approach both analytically [8, 9] and numerically $[10,11,12,13]$. In particular, fuzzy complex projective spaces, flag manifolds and toric varieties have been constructed $[14,15,16]$ and provide a wide generalization of previous examples. Important matters to be addressed are how to construct fuzzy spinor fields and the related Dirac operator. These were first constructed in [17] for the fuzzy 2-sphere; previous work aimed at generalizing this construction to other fuzzy spaces include $[18,19]$ and quantization of vector bundles was considered in [20, 21].

In the present work we construct a universal Dirac operator on fuzzy $\mathbb{C P}^{n}$ and its associated eigenspinors; whereas only $\mathbb{C P}^{2 n+1}$ admits a spin structure, $\mathbb{C P}^{n}$ admits a countably infinite number of $\operatorname{spin}^{c}$ structures [22]. We also reproduce the correct spectrum of the commutative Dirac operator up to a truncation.

In noncommutative geometry sections of the spin bundles are replaced with finitely generated projective modules over a noncommutative algebra. This definition of noncommutative sections is motivated by the Serre-Swan theorem [23] which states that the space of smooth sections of a vector bundle over a compact space $\mathrm{M}$ is a projective module of finite type over the algebra $\mathrm{C}^{\infty}(M)$ of smooth functions over $\mathrm{M}$, and any finite projective $\mathrm{C}^{\infty}(M)$-module can be realized as the module of sections of some bundle over M. In [24] we provided a construction of fuzzy vector bundles in this spirit and in the following we extend this to spinor bundles.

Section 2 contains the basic definitions and presents the multi-oscillator construction previously introduced in [24]. It also introduces appropriate 
covariant derivatives used in the definition of the Dirac operator. Section 3 is a brief review of the construction of $\operatorname{spin}^{c}$ bundles over $\mathbb{C P}^{n}$. Section 4 presents fuzzy spin bundles and the universal Dirac operator, along with its eigenspinors and a novel presentation of the spectrum and comments about the zero modes. The explicit calculation of the complete spectrum and the construction of a basis for all fuzzy spinors is left to the appendices. Finally, in section 5 we briefly discuss charge conjugation. Our conclusions and outlook are present in section 6 .

\section{Review of Multi-Oscillator Construction}

In [24] we introduced a Fock space, $\mathcal{F}^{\text {Total }}$ generated freely by the action of $n(n+1)$ oscillators $\left(a^{\dagger}\right)_{\imath}^{\alpha}=a_{\imath}^{\dagger \alpha}=\left(a_{\alpha}^{\imath}\right)^{\dagger}$ satisfying

$$
\left[a_{\alpha}^{\imath}, a_{\jmath}^{\dagger}\right]=\delta_{\jmath}^{\imath} \delta_{\alpha}^{\beta}, \quad \imath=1, \ldots, n, \quad \alpha=1, \ldots, n+1 .
$$

These oscillators, $a_{\imath}^{\dagger}{ }_{\imath}$, carry the anti-fundamental representation of $u(n+1)$ and the fundamental representation of $u(n)$. The generators of $U(n+1)$ and $U(n)$ are given by the Schwinger-Jordan construction as

$$
\begin{aligned}
\hat{J}^{\alpha}{ }_{\beta} & :=a^{\dagger^{\alpha}}{ }_{\imath} a_{\beta}^{\imath} \\
\hat{J}_{\imath}^{\jmath} & :=a^{\dagger \alpha}{ }_{\imath} a_{\alpha}^{\jmath}
\end{aligned}
$$

respectively. These include a common $U(1)$ generator, the total number operator

$$
\widehat{N}:=a_{\imath}^{\dagger} a_{\alpha}^{\imath} .
$$

So the oscillators $a_{\imath}^{\dagger^{\alpha}}$ and hence the Fock space $\mathcal{F}^{\text {Total }}$, carry a $s u(n+1) \times$ $s u(n) \times u(1)$ representation.

We also introduced composite operators $\widetilde{A}^{\alpha}, \widetilde{A}_{\alpha}^{\dagger}$ formed by using the completely antisymmetric tensor, $\epsilon_{\imath_{1} \cdots v_{n}}$, to form an $s u(n)$ singlet from the oscillators $a_{\alpha}^{\imath}$ (and dualizing the $n s u(n+1)$ indices):

$$
\widetilde{A}^{\alpha}:=\frac{1}{n !} \epsilon^{\alpha \beta_{1} \cdots \beta_{n}} \epsilon_{\imath_{1} \cdots l_{n}} a_{\beta_{1}}^{\imath_{1}} \cdots a_{\beta_{n}}^{\imath_{n}} .
$$

$\widetilde{A}_{\alpha}^{\dagger}$ generate a subspace of $\mathcal{F}^{\text {Total }}$ which we call the reduced Fock space, $\mathcal{F}$, which is a singlet under $s u(n)$. It naturally decomposes into a direct sum of subspaces, $\mathcal{F}_{L}$, associated with the eigenvalue, $L$, of the number operator on the reduced Fock space

$$
\widehat{\mathcal{N}}:=\frac{1}{n} a_{\imath}^{\dagger} a_{\alpha}^{\imath}=\frac{\widehat{N}}{n}
$$


so that

$$
\mathcal{F}=\bigoplus_{L=0}^{\infty} \mathcal{F}_{L}
$$

where $\mathcal{F}_{L}$ is spanned by $\widetilde{A}_{\alpha_{1}}^{\dagger} \cdots \widetilde{A}_{\alpha_{L}}^{\dagger} \mid 0>$.

The above identifies $\mathcal{F}$, the subspace of $\mathcal{F}^{\text {Total }}$ which carries the singlet 1 representation of $s u(n)$. In the decomposition of $\mathcal{F}^{\text {Total }}$ into irreducible representations of the algebra $s u(n+1) \times s u(n) \times u(1)$ all irreps., $\mathcal{R}$, of $u(n)$ appear with multiplicity one,

$$
\mathcal{F}^{\text {Total }}=\oplus_{\mathcal{R}} \mathcal{F}_{\mathcal{R}}
$$

For example, if $\mathcal{R}$ is the fundamental representation, $\square$, of $u(n)$ then $\mathcal{F}_{\square}=$ $\oplus_{L=0}^{\infty} \mathcal{F}_{\square}^{L}$ is given by the span of

$$
\left(a^{\dagger}\right)_{\imath}^{\beta} \mathcal{F}=\bigoplus_{L=0}^{\infty}\left(a^{\dagger}\right)_{\imath}^{\beta} \mathcal{F}^{L}
$$

(we normalise the $U(1)$ charge so that $\left(a^{\dagger}\right)_{\imath}^{\beta}$ has charge $1 / n$ and $\widetilde{A}_{\alpha}^{\dagger}$ charge 1 , then states in $\mathcal{F}_{\square}^{L}$ have charge $\left.L+\frac{1}{n}\right)$.

The composite oscillators do not satisfy the Heisenberg algebra although they do generate a closed subspace of $\mathcal{F}^{\text {Total }}$. We have shown [24] that it is possible to normalize them in such a way so that they do however satisfy the Heisenberg algebra on the reduced Fock space, $\mathcal{F}$. Define

$$
\begin{aligned}
& A^{\alpha}:=\widetilde{A}^{\alpha} \sqrt{\frac{\hat{\mathcal{N}} !}{(\widehat{\mathcal{N}}+n-1) !}} \\
& A_{\alpha}^{\dagger}:=\sqrt{\frac{\widehat{\mathcal{N}} !}{(\widehat{\mathcal{N}}+n-1) !}} \widetilde{A}_{\alpha}^{\dagger} .
\end{aligned}
$$

Then denoting elements of $\mathcal{F}_{L}$ by

$$
|\boldsymbol{\alpha}\rangle:=\frac{1}{L !} A_{\alpha_{1}}^{\dagger} \cdots A_{\alpha_{L}}^{\dagger}|0\rangle
$$

or

$$
|\widetilde{\boldsymbol{\alpha}}\rangle:=\frac{1}{L !} \widetilde{A}_{\alpha_{1}}^{\dagger} \cdots \widetilde{A}_{\alpha_{L}}^{\dagger}|0\rangle
$$

we have

$$
\left[A^{\alpha}, A_{\beta}^{\dagger}\right]=\delta_{\beta}^{\alpha}
$$

on such elements. 
It is then easy to see that the standard Heisenberg algebra / Fock space relations hold

$$
\begin{aligned}
& \langle\boldsymbol{\alpha} \mid \boldsymbol{\beta}\rangle=S_{\boldsymbol{\beta}}^{\boldsymbol{\alpha}}:=\frac{1}{L !} \delta_{\left\{\beta_{1}\right.}^{\alpha_{1}} \cdots \delta_{\left.\beta_{L}\right\}}^{\alpha_{L}} \\
& \widehat{\mathcal{N}}_{A}|\boldsymbol{\alpha}\rangle:=A_{\beta}^{\dagger} A^{\beta}|\boldsymbol{\alpha}\rangle=\widehat{\mathcal{N}}|\boldsymbol{\alpha}\rangle=L|\boldsymbol{\alpha}\rangle
\end{aligned}
$$

though $\widehat{\mathcal{N}}_{A} \neq \widehat{\mathcal{N}}$ on the full Fock space generated by $a_{\imath}^{\dagger \alpha}$, they are only equal on the reduced Fock space.

We can also realise the generators of $S U(n+1)$ in the usual way

$$
\hat{L}_{a}:=A_{\alpha}^{\dagger}\left(\frac{\lambda_{a}}{2}\right)^{\alpha}{ }_{\beta} A^{\beta}
$$

On the reduced Fock space these can be shown to be equivalent to the $S U(n+$ 1) generators $\hat{J}_{a}:=\left(\frac{\bar{\lambda}_{a}}{2}\right)_{\alpha}{ }^{\beta} \hat{J}^{\alpha}{ }_{\beta}$ written in term of the oscillators $a_{\alpha}^{\imath}$ and $\left(a^{\dagger}\right)_{\imath}^{\alpha}$

$$
\hat{J}_{a}|\boldsymbol{\alpha}\rangle=\hat{L}_{a}|\boldsymbol{\alpha}\rangle
$$

though $\hat{J}_{a} \neq \hat{L}_{a}$ on $\mathcal{F}^{\text {Total }}$.

The $s u(n)$ singlet nature of $A_{\alpha}^{\dagger}$ is shown by

$$
\hat{J}_{\imath}{ }^{\jmath}|\boldsymbol{\alpha}\rangle=\delta_{\imath}^{\jmath} \widehat{\mathcal{N}}|\boldsymbol{\alpha}\rangle=L \delta_{\imath}^{\jmath}|\boldsymbol{\alpha}\rangle
$$

and

$$
\left(a^{\dagger}\right)_{\imath}^{\alpha} A_{\alpha}^{\dagger}=0 \text {. }
$$

The action of an $s u(n+1)$ generator on a singlet state is given by

$$
\hat{J}_{\beta}^{\gamma}|\boldsymbol{\alpha}\rangle=L \delta_{\beta}^{\gamma}|\boldsymbol{\alpha}\rangle-\frac{1}{\sqrt{L !}} \sum_{k=1}^{L} \delta_{\alpha_{k}}^{\gamma} A_{\beta}^{\dagger} A_{\alpha_{1}}^{\dagger} \cdots A_{\alpha_{k-1}}^{\dagger} A_{\alpha_{k+1}}^{\dagger} \cdots A_{\alpha_{L}}^{\dagger} \mid 0>\text {. }
$$

$\mathcal{F}_{L}$ is a left module for the noncommutative algebra of functions, $\mathcal{A}_{L}:=$ $\mathcal{F}_{L} \otimes \mathcal{F}_{L}^{*}$ and a basis is $|\boldsymbol{\alpha}\rangle\langle\boldsymbol{\beta}|$. Since the dimension of $\mathcal{F}_{L}$ is

$$
\operatorname{dim}(\overbrace{\square \square}^{L})_{s u(n+1)}=\frac{(L+n) !}{L ! n !}=: d_{n}(L),
$$

$\mathcal{A}_{L}$ is isomorphic to $\mathrm{Mat}_{d_{n}(L)}$ and is the space of linear mappings from $\mathcal{F}_{L}$ to $\mathcal{F}_{L}$. The dimension can also be seen by taking the trace of the identity matrix,

$$
\operatorname{Tr}(\mathbf{1})=\operatorname{Tr}(|\boldsymbol{\alpha}\rangle\langle\boldsymbol{\alpha}|)=\langle\boldsymbol{\alpha} \mid \boldsymbol{\alpha}\rangle=d_{n}(L) .
$$

More generally we can extend the construction to non-square matrices and consider $\mathcal{F}_{L} \otimes \mathcal{F}_{L^{\prime}}^{*}$ as the space of linear mappings from $\mathcal{F}_{L^{\prime}}$ to $\mathcal{F}_{L}$. As 
shown in [24] these describe noncommutative equivariant line bundles over $\mathbb{C P}^{n}=S U(n+1) / U(n)$ : elements of the set of such maps are carrier spaces for $S U(n+1)$, invariant under $S U(n)$ and have a $U(1)$ charge proportional to $q:=L-L^{\prime}$. Such matrices form a left $\operatorname{Mat}_{d_{n}(L)}$ module and a right $\operatorname{Mat}_{d_{n}\left(L^{\prime}\right)}$ module. We will also consider $\mathcal{F}_{L} \otimes \mathcal{F}_{\mathcal{R}}$, corresponding to equivariant vector bundles over $\mathbb{C} \mathbb{P}^{n}$, in the next section, but for the remainder of this section we focus on line bundles $\mathcal{F}_{L} \otimes \mathcal{F}_{L^{\prime}}^{*}$.

A general $d_{n}(L) \times d_{n}\left(L^{\prime}\right)$ matrix $\mathbf{M} \in \mathcal{F}_{L} \otimes \mathcal{F}_{L^{\prime}}^{*}$ takes the form

$$
\mathbf{M}=M_{\boldsymbol{\beta}}^{\boldsymbol{\alpha}}|\boldsymbol{\alpha}\rangle\langle\boldsymbol{\beta}|
$$

where $M^{\boldsymbol{\alpha}} \boldsymbol{\beta}_{\boldsymbol{\beta}}=M_{\beta_{1} \cdots \beta_{L^{\prime}}}^{\alpha_{1} \cdots \alpha_{L}}$ are complex co-efficients. The action of $s u(n+1)$ on $\mathcal{F}_{L} \otimes \mathcal{F}_{L^{\prime}}^{*}$ is $\left(\hat{L}_{a} \mathcal{F}_{L} \otimes \mathcal{F}_{L^{\prime}}^{*}\right)+\left(\mathcal{F}_{L} \otimes \mathcal{F}_{L^{\prime}}^{*}\left(-\hat{L}_{a}\right)\right)$; this leads us to define

$$
\begin{aligned}
\hat{\mathcal{L}}_{a} \mathbf{M} & :=\left(\hat{L}_{a}\right)^{\mathrm{L}} \mathbf{M}-\left(\hat{L}_{a}\right)^{\mathrm{R}} \mathbf{M}, \\
& =\hat{L}_{a} \mathbf{M}-\mathbf{M} \hat{L}_{a}
\end{aligned}
$$

where the superscripts ${ }^{\mathrm{L}}$ and ${ }^{\mathrm{R}}$ indicate left and right action respectively, and the operators $\hat{\mathcal{L}}_{a}$ can be seen as right invariant vector fields induced from the left action of $S U(n+1)$ on $\mathbb{C P}^{n}$, they satisfy the $s u(n+1)$ commutation relations.

A Laplacian for $\mathcal{F}_{L} \otimes \mathcal{F}_{L^{\prime}}$ can be immediately constructed from left actions

$$
\Delta \mathbf{M}=\hat{\mathcal{L}}_{a} \hat{\mathcal{L}}_{a} \mathbf{M}
$$

This is just the $s u(n+1)$ quadratic Casimir operator corresponding to the appropriate representation, explicitly

$$
\Delta=\left(\hat{L}_{a} \hat{L}_{a}\right)^{\mathrm{L}} \otimes \mathbf{1}+\mathbf{1} \otimes\left(\hat{L}_{a} \hat{L}_{a}\right)^{\mathrm{R}}-2 \hat{L}_{a}^{\mathrm{L}} \otimes \hat{L}_{a}^{\mathrm{R}} .
$$

It was also shown in [24] that a diagonal basis for $\mathcal{F}_{L} \otimes \mathcal{F}_{L^{\prime}}$ can be realised as the set of (here un-normalized) polarization tensors which are eigenstates of the Laplacian, $\mathbf{D}_{\boldsymbol{\alpha}_{l+q}} \boldsymbol{\beta}_{l} \in \mathcal{F}_{L} \otimes \mathcal{F}_{L^{\prime}}^{*}$, given by

$$
\mathbf{D}_{\boldsymbol{\alpha}_{l+q}} \boldsymbol{\beta}_{l}=\mathcal{P}_{\boldsymbol{\alpha}_{l+q}, \boldsymbol{\sigma}_{l}} \boldsymbol{\beta}_{l}, \boldsymbol{\tau}_{l+q}\left|\boldsymbol{\tau}_{l+q}, \boldsymbol{\gamma}_{L^{\prime}-l}\right\rangle\left\langle\boldsymbol{\sigma}_{l}, \boldsymbol{\gamma}_{L^{\prime}-l}\right|
$$

where $\mathcal{P}_{\boldsymbol{\alpha}_{l+q}, \boldsymbol{\sigma}_{l}} \boldsymbol{\beta}_{l}, \boldsymbol{\tau}_{l+q}$ is the projector that removes all traces associated with contractions of the free indices of $\mathcal{F}_{L}$ and $\mathcal{F}_{L^{\prime}}^{*} ; \boldsymbol{\alpha}_{l}$ indicates $l$ free indices $\alpha_{1} \cdots \alpha_{l}$. We write a non-square eigenmatrix of the Laplacian, $\boldsymbol{\Phi}_{l_{\mathrm{L}}, l_{\mathrm{R}}}$, as

$$
\Phi_{l_{\mathrm{L}}, l_{\mathrm{R}}}=\phi_{\boldsymbol{\beta}}^{\boldsymbol{\alpha}}\left|\boldsymbol{\alpha}_{l_{\mathrm{L}}}, \gamma\right\rangle\left\langle\boldsymbol{\gamma}, \boldsymbol{\beta}_{l_{\mathrm{R}}}\right|
$$

where the coefficients $\phi_{\boldsymbol{\beta}}^{\boldsymbol{\alpha}}$ are traceless under contractions of any pair of upper and lower indices and here $l_{\mathrm{L}}=l_{\mathrm{R}}+q$. 
The spectrum of the Laplacian can be obtained from its action on $\boldsymbol{\Phi}_{l_{\mathrm{L}}, l_{\mathrm{R}}}$

$$
\Delta \boldsymbol{\Phi}_{l_{\mathrm{L}}, l_{\mathrm{R}}}=\frac{1}{2}\left(l_{\mathrm{L}}\left(l_{\mathrm{R}}+n\right)+l_{\mathrm{R}}\left(l_{\mathrm{L}}+n\right)+\frac{n\left(l_{\mathrm{L}}-l_{\mathrm{R}}\right)^{2}}{n+1}\right) \boldsymbol{\Phi}_{l_{\mathrm{L}}, l_{\mathrm{R}}} .
$$

Polarization tensors (28) form a complete orthogonal basis for $\mathcal{F}_{L} \otimes \mathcal{F}_{L^{\prime}}$ under the trace inner product:

$$
\langle\mathbf{M}, \mathbf{N}\rangle:=\operatorname{Tr}\left(\mathbf{M}^{\dagger} \mathbf{N}\right)
$$

The matrices $\boldsymbol{\Phi}_{l_{\mathrm{L}}, l_{\mathrm{R}}}$ represent sections of line bundles, at the fuzzy level. Let $\mathbf{L}$ denote the tautological line-bundle over $\mathbb{C P}^{n}$ in the exact sequence

$$
0 \rightarrow \mathbf{L} \rightarrow \mathbf{V} \rightarrow \mathbf{F} \rightarrow 0
$$

where $\mathbf{V}=\mathbb{C P}^{n} \otimes \mathbb{C}^{n+1}$ is the trivial rank $n+1$ complex bundle over $\mathbb{C P}^{n}$ and $\mathbf{F}$ is the rank $n$ bundle over $\mathbb{C P}^{n}$ induced by the above sequence. Then $\mathbf{L}$ has first Chern number $C_{1}(\mathbf{L})=-1$ and $\boldsymbol{\Phi}_{l_{\mathrm{L}}, l_{\mathrm{R}}}$ is a fuzzy section of $\mathbf{L}^{l_{\mathrm{L}}-l_{\mathrm{R}}}$. Monopole bundles over fuzzy complex projective spaces were constructed, in terms of projective modules, in [25].

\subsection{Covariant Derivatives}

In [24] we also introduced operators ${ }^{1}$ corresponding to right action of the compliment of the $u(n)$ subalgebra in $s u(n+1)$ on equivariant vector bundles over $\mathbb{C P}^{n}, \hat{K}_{\imath}, \hat{K}_{\bar{\imath}}$, which act as covariant derivatives on the spaces of noncommutative functions $\mathcal{A}_{L}=\mathcal{F}_{L} \otimes \mathcal{F}_{L}^{*}$ and noncommutative line bundles $\mathcal{F}_{L} \otimes \mathcal{F}_{L-q}^{*}$

$$
\begin{array}{llll}
\hat{K}_{\imath}:=\left(A_{\alpha}^{\dagger}\right)^{\mathrm{L}}\left(\left(a^{\dagger}\right)_{\imath}^{\alpha}\right)^{\mathrm{R}} & : \mathcal{F}_{L} \otimes \mathcal{F}_{L^{\prime}}^{*} \longmapsto \mathcal{F}_{L+1} \otimes \mathcal{F}_{L^{\prime}, \imath}^{*} \\
\hat{K}_{\bar{\imath}}:=\left(A^{\alpha}\right)^{\mathrm{L}}\left(a_{\alpha}^{\imath}\right)^{\mathrm{R}} & : \mathcal{F}_{L} \otimes \mathcal{F}_{L^{\prime}}^{*} \longmapsto \mathcal{F}_{L-1} \otimes \mathcal{F}_{L^{\prime}, \bar{\imath}}^{*}
\end{array}
$$

where we have denoted the subspace of Fock space spanned by vectors of the form

$$
\left(a^{\dagger}\right)_{\imath}^{\alpha} A_{\alpha_{1}}^{\dagger} \ldots A_{\alpha_{L}}^{\dagger}|0\rangle
$$

by $\mathcal{F}_{L, \imath}=\mathcal{F}_{L}^{\bar{\imath}}$. The remainder of the left invariant vector fields generating $U(n)$ acting on $\mathbb{C P}^{n}$ are given by $\left[\hat{K}_{\imath}, \hat{K}_{\bar{\jmath}}\right]$. For a matrix $\mathbf{M}_{\mathcal{R}} \in \mathcal{F}_{L} \otimes \mathcal{F}_{\mathcal{R}}^{*}$ we find

$$
\left[\hat{K}_{\imath}, \hat{K}_{\bar{\jmath}}\right] \mathbf{M}_{\mathcal{R}}=-\mathbf{M}_{\mathcal{R}}\left(\hat{J}_{\imath}{ }^{\jmath}-\delta_{\imath}^{\jmath} \hat{\mathcal{N}}\right)+2 \delta_{\imath}^{\jmath} \hat{K}_{0} \mathbf{M}_{\mathcal{R}}
$$

\footnotetext{
${ }^{1}$ For $\mathbb{C P}^{1}$ these operators were already given by Grosse et al. [26].
} 
where $\hat{K}_{0}:=\frac{1}{2}\left(\hat{\mathcal{N}}_{A}^{\mathrm{L}}-\hat{\mathcal{N}}^{\mathrm{R}}\right)$, and we easily see that $\mathcal{F}_{L} \otimes \mathcal{F}_{L}^{*}$ is invariant under the right action of $U(n)$ as expected. The Laplacian acting over $\mathbf{M}_{\mathcal{R}}$ is naturally the quadratic $s u(n+1)$ Casimir on $\mathcal{F}_{L} \otimes \mathcal{F}_{\mathcal{R}}^{*}$

$$
\Delta \mathbf{M}_{\mathcal{R}}=\left(\hat{L}_{a}^{\mathrm{L}}-\hat{J}_{a}^{\mathrm{R}}\right)^{2} \mathbf{M}_{\mathcal{R}}
$$

and, when $\mathcal{R}$ is the symmetric combination of $L^{\prime}$ anti-fundamental representations of $s u(n+1) \overbrace{\text { ind }}^{L^{\prime}}\}^{n}$. The Laplacian can be constructed in terms of right actions as

$$
\Delta_{K}=\frac{1}{2}\left(\hat{K}_{\imath} \hat{K}_{\bar{\imath}}+\hat{K}_{\bar{\imath}} \hat{K}_{\imath}\right)+\frac{2 n}{n+1} \hat{K}_{0}^{2} .
$$

On eignematrices $\Phi_{l_{\mathrm{L}}, l_{\mathrm{R}}}, \Delta_{K}$ coincides with (27) having the same eigenvectors and spectrum (30) as can be verified from the relations

$$
\hat{K}_{\imath} \hat{K}_{\bar{\imath}} \boldsymbol{\Phi}_{l_{\mathrm{L}}, l_{\mathrm{R}}}=l_{\mathrm{L}}\left(l_{\mathrm{R}}+n\right) \boldsymbol{\Phi}_{l_{\mathrm{L}}, l_{\mathrm{R}}}
$$

and

$$
\hat{K}_{\bar{\imath}} \hat{K}_{\imath} \boldsymbol{\Phi}_{l_{\mathrm{L}}, l_{\mathrm{R}}}=l_{\mathrm{R}}\left(l_{\mathrm{L}}+n\right) \boldsymbol{\Phi}_{l_{\mathrm{L}}, l_{\mathrm{R}}},
$$

with $\hat{K}_{0}=\left(l_{\mathrm{L}}-l_{\mathrm{R}}\right) / 2$.

\section{Spinors}

On a $2 n$ dimensional real manifold, with a Euclidean metric, the $\Gamma$-matrices can be chosen to satisfy

$$
\left\{\Gamma^{\mu}, \Gamma^{\nu}\right\}=-2 \delta^{\mu \nu}, \quad \mu, \nu=1, \ldots 2 n
$$

in an orthonormal basis and to be anti-Hermitian, $\left(\Gamma^{\mu}\right)^{\dagger}=-\Gamma^{\mu}$. On an $n$-complex dimensional Kähler manifold, such as $\mathbb{C P}^{n}$, we can define $\gamma^{2}=$ $\left(-i \Gamma^{\imath}+\Gamma^{\imath+n}\right) / 2$, with $\imath=1, \ldots, n$. The Dirac algebra in this basis is

$$
\begin{aligned}
& \left\{\gamma^{\imath}, \gamma^{\jmath}\right\}=\left\{\gamma^{\bar{\imath}}, \gamma^{\bar{\jmath}}\right\}=0 \\
& \left\{\gamma^{\imath}, \gamma^{\bar{\jmath}}\right\}=\delta^{\imath \bar{\jmath}},
\end{aligned}
$$

where $\gamma^{\bar{\imath}}=\left(\gamma^{2}\right)^{\dagger}$. One can think of $\gamma^{\bar{\imath}}$ as fermionic creation operators and $\gamma^{2}$ as the Hermitian conjugate fermionic annihilation operators. The spinors, on which the $\gamma$-matrices act, can be constructed by acting on a Clifford vacuum $|\Omega\rangle$ defined by $\gamma^{2}|\Omega\rangle=0$. A general spinor field then has an expansion [27]

$$
\Psi=\psi_{0}|\Omega\rangle+\psi_{\bar{\imath}} \gamma^{\bar{\imath}}|\Omega\rangle+\frac{1}{2 !} \psi_{\overline{\bar{\jmath}}} \gamma^{\bar{i}} \gamma^{\bar{\jmath}}|\Omega\rangle+\cdots+\psi_{\bar{\imath}_{1} \bar{z}_{2} \cdots \bar{\tau}_{n}} \gamma^{\bar{\nu}_{1}} \gamma^{\bar{\tau}_{2}} \cdots \gamma^{\bar{\tau}_{n}}|\Omega\rangle .
$$


The chirality operator is $\Gamma:=\prod_{\imath=1}^{n}\left[\gamma^{\imath}, \gamma^{\bar{\imath}}\right]$ satisfying $\left\{\Gamma, \gamma^{\imath}\right\}=\left\{\Gamma, \gamma^{\bar{\imath}}\right\}=0$ and $\Gamma^{2}=1$. It has the effect of changing the sign of the terms in $\Psi$ with an odd number of $\gamma$-matrices.

Because the holonomy group is $U(n)$, we do not, in general, expect $\psi_{0}$ to be neutral under the $U(1)$ part of the $U(n)$-valued spin connection, i.e. $\psi_{0}$ is a charged scalar field.

Let $\wedge^{0, k} \overline{T^{*} \mathbb{C P}^{n}}$ be the bundle of $(0, k)$-forms and

$$
\wedge^{0, *} T \mathbb{C P}^{n}=\bigoplus_{k=0}^{n} \wedge^{0, k} \overline{T^{*} \mathbb{C} \mathbb{P}^{n}}
$$

be the bundle of linear combinations of $(0, k)$-forms. Then the spin bundle $S\left(\mathbb{C P}^{n}\right)$ can be defined by $[22]$

$$
S\left(\mathbb{C} \mathbb{P}^{n}\right):=\wedge^{0, *} T \mathbb{C} \mathbb{P}^{n} \otimes \mathbf{L}^{\frac{n+1}{2}} .
$$

It only exists for odd $n$ as can be seen from the power of the line bundle $\mathbf{L}$, reflecting the fact that only $\mathbb{C P}^{n}$ with odd $n$ has spin structure. It is possible to overcome the odd $n$ restriction by considering $\operatorname{spin}^{c}$ structures. We simply tensor with different integer powers of $\mathbf{L}$ :

$$
S_{q}\left(\mathbb{C} P^{n}\right):=\wedge^{0, *} T \mathbb{C P} \mathbb{P}^{n} \otimes \mathbf{L}^{q}, \quad q \in \mathbb{Z} .
$$

These bundles exist for all $n$ and give general $\operatorname{spin}^{c}$ bundles - the case $q=0$, where $\psi_{0}$ in (43) has no $U(1)$ charge and so is just a function, is canonical spin ${ }^{c}$. Sections of $S_{q}\left(\mathbb{C P}^{n}\right)$ can be viewed as linear combinations of $(0, k)$-forms, each with charge $q$, and we shall denote the space of charged $(0, k)$-forms by $\Lambda^{0, k}\left(\mathbb{C} \mathbb{P}^{n}, q\right)$. Note that for canonical $\operatorname{spin}^{c}, S_{0}\left(\mathbb{C P}^{n}\right)$ is just the bundle of linear combinations of $(0, k)$-forms, with $q=0$, while ordinary spinors, i.e. sections of $S\left(\mathbb{C P}^{n}\right)$ in $(45)$ when $n$ is odd, can be identified with linear combinations of $(0, k)$-forms carrying a $U(1)$ charge $(n+1) / 2$. For $S U(n)$ holonomy such complications do not occur and ordinary spinors are neutral $(0, k)$-forms.

The Dirac operator

$$
\not D=\gamma^{\bar{\imath}} D_{\bar{\imath}}+\gamma^{\imath} D_{\imath}=\overline{\mathcal{D}}+\overline{\mathcal{D}}^{\dagger},
$$

where $\overline{\mathcal{D}}:=\gamma^{\bar{\imath}} D_{\bar{\imath}}$ and $\overline{\mathcal{D}}^{\dagger}:=\gamma^{\imath} D_{\imath}$ its adjoint, acts on sections of $S_{q}\left(\mathbb{C P}^{n}\right)$ and can be constructed once the relevant co-variant derivatives, $D_{\bar{\imath}}$ and $D_{\imath}$, are known. The bundles (46) are holomorphic in the sense that

$$
F_{\imath \jmath}=\left[D_{\imath}, D_{\jmath}\right]=0, \quad F_{\bar{\imath} \bar{\jmath}}=\left[D_{\bar{\imath}}, D_{\bar{\jmath}}\right]=0 .
$$




\section{Noncommutative Spinors ${ }^{2}$}

In section 2.1 we defined covariant derivative operators, $K_{\imath}$ and $K_{\bar{\imath}}$, acting on fuzzy $\mathbb{C P}^{n}$ and we shall now show that these are indeed the correct objects to use in the fuzzy Dirac equation. We propose

$$
\not D=\overline{\mathcal{D}}+\overline{\mathcal{D}}^{\dagger}:=\gamma^{\bar{\imath}} \hat{K}_{\bar{\imath}}+\gamma^{\imath} \hat{K}_{\imath}
$$

as a noncommutative massless Dirac operator. We shall show that this has the same spectrum as the known continuum cases cut-off at a finite level. The Dirac operator (49) clearly anti-commutes with the chirality operator, $\{\not D, \Gamma\}=0$.

A detailed discussion of the complete spectrum of (49) with an explicit construction of all eigenspinors together with their corresponding eigenvalues and degeneracies is given in two appendices, where it is shown that the nonzero eigenvalues $\lambda$ are labelled by three integers $q, l$ and $k$, with $l=0, \ldots, L$ and $k=0, \ldots, n-1$ constrained by $l+q \geq k+1$, and are given by

$$
\lambda= \pm \sqrt{(l+q)(l-k+n)},
$$

with degeneracies

$$
g_{\lambda}=\frac{(l+n) !(l+q-k-1+n) !(2 l+q-k+n)}{l ! k ! n !(l+q-k-1) !(n-k-1) !(l+n-k)(l+q)}
$$

For the moment, for simplicity, we shall motivate the choice (49) with a discussion of the zero modes, explicitly constructing all such modes for any $n$ and proving that everything is consistent with known results.

The noncommutative version of sections of the line bundles $\mathbf{L}^{q}, q \geqslant 0$ have already been identified: they are just the bi-modules

$$
\mathcal{F}_{L^{\prime}+q} \otimes \mathcal{F}_{L^{\prime}}^{*}
$$

and have $U(1)$ charge $q$ as measured by $2 \hat{K}_{0}$ (in the conventions adopted here the charge is the opposite of the first Chern number $\left.C_{1}\left(\mathbf{L}^{q}\right)=-q\right)$. Similarly the noncommutative counterpart of sections of the line bundles $\mathbf{L}^{q}$ with $q<0$ are described by the bi-modules

$$
\mathcal{F}_{L} \otimes \mathcal{F}_{L+|q|}^{*} .
$$

\footnotetext{
${ }^{2}$ We shall call elements of the projective modules corresponding to sections of the spin ${ }^{\mathrm{c}}$ bundle(s) over fuzzy $\mathbb{C P}^{n}$ "noncommutative spinors", ignoring the question of a wedge product for their charged $(0, k)$ form constituents. We will not give a wedge product but work only with modules.
} 
Specific elements of these line-bundles are zero-modes of the Dirac operator.

The Atiyah-Singer index, $\nu$, for zero modes of the Dirac operator on sections of the bundle $S_{q}\left(\mathbb{C P}^{n}\right) \approx S_{0}\left(\mathbb{C P}^{n}\right) \otimes \mathbf{L}^{q}$, where $q \in \mathbf{Z}$, is [28]

$$
\nu=\frac{(1-q) \cdots(n-q)}{n !} .
$$

For $q \leq 0$ this is the same as the dimension of the symmetric representation of $s u(n+1)$ with a Young tableau consisting of a row of $|q|$ fundamental representations:

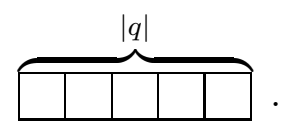

For $q \geq(n+1)$ the magnitude $|\nu|$ of the index (54) is the same as the dimension of the representation consisting of $q-n-1$ symmetrised antifundamentals of $s u(n+1)$,

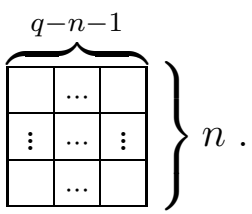

For $0<q<n+1$ the index is zero. For odd $n, \nu$ is positive for $q \leq 0$ and negative for $q \geq n+1$ while, for even $n, \nu$ is always positive.

These Young tableaux reflect the symmetries of the wave-functions corresponding to the zero-modes. Our proposal for the Dirac operator is

$$
\not D=\left(A^{\alpha}\right)^{\mathrm{L}}\left(a_{\alpha}^{\imath}\right)^{\mathrm{R}} \gamma^{\bar{\imath}}+\left(A_{\alpha}^{\dagger}\right)^{\mathrm{L}}\left(a_{\imath}^{\dagger \alpha}\right)^{\mathrm{R}} \gamma^{\imath}
$$

Zero modes for $q \leq 0$ are

$$
\boldsymbol{\psi}_{q}^{\boldsymbol{\alpha}}=|0><0| A^{\alpha_{1}} \cdots A^{\alpha_{|q|}} \otimes \mid \Omega>,
$$

while the zero modes for $q \geq n+1$ are

$$
\widetilde{\boldsymbol{\psi}}_{q, \boldsymbol{\alpha}}=A_{\alpha_{1}}^{\dagger} \cdots A_{\alpha_{q-n-1}}^{\dagger}|0><0| \otimes \gamma^{\overline{1}} \cdots \gamma^{\bar{n}} \mid \Omega>
$$

These are the smallest possible expressions of the zero modes for a given $q$ - they can be embedded into bigger matrices using

$$
\left|\boldsymbol{\mu}_{L}><\boldsymbol{\mu}_{L}\right| A^{\alpha_{1}} \cdots A^{\alpha_{|q|}} \otimes \mid \Omega>
$$

and

$$
A_{\alpha_{1}}^{\dagger} \cdots A_{\alpha_{q-n-1}}^{\dagger}\left|\boldsymbol{\mu}_{L}><\boldsymbol{\mu}_{L}\right| \otimes \gamma^{\overline{1}} \cdots \gamma^{\bar{n}} \mid \Omega>
$$


respectively.

Given that we have assigned the $U(1)$ charges $Q\left(A_{\alpha}^{\dagger}\right)=1$ to $A_{\alpha}^{\dagger}$, and $Q\left(a_{\imath}^{\dagger \alpha}\right)=1 / n$ to $a_{\imath}^{\dagger \alpha}$, demanding that the Dirac operator commutes with the charge operator requires assigning the charge $Q\left(\gamma^{\bar{\imath}}\right)=(n+1) / n$ to the $\gamma$ matrices. The vacuum must then be charged, $Q(\mid \Omega>)=v$, and following the arguments in [27], $Q\left(\gamma^{\overline{1}} \cdots \gamma^{\bar{n}} \mid \Omega>\right)=n+1+v=-v$ so $Q(\mid \Omega>)=-(n+1) / 2$ in the conventions used here. The $U(1)$ charge of the states in (60) and (61) is then $q-\frac{n+1}{2}$, consistent with the statement that Dirac spinors (with $q=(n+1) / 2$ for odd $n)$ have zero charge. These charge assignments can be obtained from the charge operator

$$
\widehat{Q}=\left(A_{\alpha}^{\dagger} A^{\alpha}\right)^{\mathrm{L}}-\frac{1}{n}\left(\left(a^{\dagger}\right)_{\alpha}^{\imath} a_{\imath}^{\alpha}\right)^{\mathrm{R}}-\frac{n+1}{2 n}\left[\gamma^{\imath}, \gamma^{\bar{\imath}}\right]
$$

acting on the states (60) and (61) above and (63) below. ${ }^{3}$ In all cases the total charge is $Q=q-\frac{n+1}{2}$, the last term being the contribution from the Clifford vacuum $\mid \Omega>$.

In terms of coherent state representations the wave-functions (58) and (59) are symmetric monomials, of order $|q|$ in $z^{\alpha}$ and of order $q-n-1$ in $\bar{z}_{\alpha}$ respectively. These form a basis for all zero-modes of equivariant line bundles. The family of zero modes (58) and (59) transform as irreps. of $S U(n+1)$, (55) and (56) respectively, whose dimensions agree with the index (54), up to a sign. For a given equivariant line bundle all zero modes are of the same chirality. In the coherent state representation, the most general zeromode is a linear superposition of the respective monomials in homogeneous co-ordinates $z^{\alpha}$ and $\bar{z}_{\alpha}$.

This provides strong support for the form (49) for a "universal" Dirac operator acting on all $S_{q}\left(\mathbb{C P}^{n}\right)$. A general spinor on fuzzy $\mathbb{C P}^{n}$ requires replacing the co-efficients $\psi_{\bar{\imath}_{1} \cdots \bar{\imath}_{k}}$ in (43) with Fock space operators,

$$
\Psi=\sum_{k=0}^{n} \frac{1}{k !} \boldsymbol{\psi}_{\bar{\imath}_{1} \cdots \bar{\imath}_{k}} \gamma^{\bar{\imath}_{1}} \cdots \gamma^{\bar{\imath}_{k}}|\Omega\rangle
$$

where $\boldsymbol{\psi}_{\bar{\imath}_{1} \cdots \bar{\imath}_{k}}$ are linear combinations of the operators

$$
\boldsymbol{\psi}_{\boldsymbol{\alpha}}^{k \boldsymbol{\beta}}=A_{\alpha_{1}}^{\dagger} \cdots A_{\alpha_{L+q-k}}^{\dagger}|0><0| A^{\beta_{1}} \cdots A^{\beta_{L}} a_{\alpha_{L+q-k+1}}^{\left[l_{1}\right.} \cdots a_{\alpha_{L+q}}^{\left.\imath_{k}\right]} .
$$

A full discussion of all eigenstates, non-zero eigenvalues (50) and the construction of the corresponding eigenstates is somewhat technical and is left to an appendix.

\footnotetext{
${ }^{3}$ Note that $q \neq L^{\prime}-L$ in (61), because the $\gamma$-matrices carry charge. From here on we drop the distinction between $L$ and $L^{\prime}$ adopted up till now - the size of the matrices should be clear from the context.
} 


\section{Charge Conjugation}

First recall standard results in ordinary commutative continuum space, e.g. [29]. The charge conjugation matrix is required to satisfy

$$
\left(\Gamma^{\mu}\right)^{T}= \pm C \Gamma^{\mu} C^{-1}, \quad \mu=1, \ldots, 2 n
$$

where, in $2 n$ dimensions, either sign is possible for a given $n$. Write the usual continuum Dirac operator in flat space as

$$
i \not \partial=i \Gamma^{\mu} \partial_{\mu} .
$$

We choose signature $(-, \ldots,-)$ and use the Clifford algebra (40).

In our conventions $\left(\Gamma^{\mu}\right)^{\dagger}=-\Gamma^{\mu}$ are anti-Hermitian, $\gamma^{\imath}=\left(-i \Gamma^{\imath}+\Gamma^{2+n}\right) / 2$ and the flat space Dirac operator is, using complex co-ordinates,

$$
i \not \partial=\gamma^{\imath} \partial_{\imath}+\gamma^{\bar{\imath}} \partial_{\bar{\imath}} \text {. }
$$

For example for $n=1$ we can take $\Gamma^{1}=i \sigma_{1}$ and $\Gamma^{2}=i \sigma_{2}$, with $\sigma_{1}$ and $\sigma_{2}$ Pauli matrices, and then $\gamma^{\imath}=\sigma_{+}$is real.

We use the same conventions in curved space, with $\Gamma^{\mu}$ satisfying (40) in an orthonormal basis. In general we can choose $\Gamma^{\imath}$ to be symmetric and $\Gamma^{\imath+n}$ to be anti-symmetric so $\gamma^{\imath}$ are real, with

$$
\left(\gamma^{\imath}\right)^{\dagger}=\gamma^{\bar{\imath}}=\left(\gamma^{\imath}\right)^{T} \quad \Rightarrow \quad C \gamma^{\imath} C^{-1}= \pm \gamma^{\bar{\imath}}
$$

and the charge conjugation matrix must satisfy

$$
\left[C, \Gamma^{i}\right]=0, \quad\left\{C, \Gamma^{i+n}\right\}=0
$$

for the upper sign and

$$
\left\{C, \Gamma^{i}\right\}=0, \quad\left[C, \Gamma^{i+n}\right]=0
$$

for the lower sign. So $C$ can be chosen to be

$$
C=C_{+}:= \begin{cases}\left(\gamma^{\overline{1}}+\gamma^{1}\right) \cdots\left(\gamma^{\bar{n}}+\gamma^{n}\right)=\Gamma^{1} \cdots \Gamma^{n}, & n \text { odd } \\ \left(\gamma^{\overline{1}}-\gamma^{1}\right) \cdots\left(\gamma^{\bar{n}}-\gamma^{n}\right)=\Gamma^{1+n} \cdots \Gamma^{2 n}, & n \text { even }\end{cases}
$$

for the upper sign in (68) and

$$
C=C_{-}:= \begin{cases}\left(\gamma^{\overline{1}}-\gamma^{1}\right) \cdots\left(\gamma^{\bar{n}}-\gamma^{n}\right)=-\Gamma^{1+n} \cdots \Gamma^{2 n}, & n \text { odd } \\ \left(\gamma^{\overline{1}}+\gamma^{1}\right) \cdots\left(\gamma^{\bar{n}}+\gamma^{n}\right)=\Gamma^{1} \cdots \Gamma^{n}, & n \text { even }\end{cases}
$$


for the lower sign. In general

$$
C_{ \pm}=\left(\gamma^{\overline{1}} \mp(-1)^{n} \gamma^{1}\right) \cdots\left(\gamma^{\bar{n}} \mp(-1)^{n} \gamma^{n}\right) .
$$

These satisfy $C_{ \pm}^{\dagger} C_{ \pm}=1, C_{ \pm}^{T}=(-1)^{n(n \mp 1) / 2} C_{ \pm}$and $C_{ \pm} \Gamma=(-1)^{n} \Gamma C_{ \pm}$.

In the basis we are using $\gamma^{\bar{\imath}}$ are real and $\mid \Omega>$ can be chosen to be real, $\mid \Omega>=\left(\mid \Omega>^{*}\right)$, so

$$
\begin{aligned}
C_{ \pm}\left(\gamma^{\bar{\iota}_{1}} \cdots \gamma^{\bar{\imath}_{k}} \mid \Omega>\right) & \left.=\left(C_{ \pm} \gamma^{\bar{\imath}_{1}} C_{ \pm}^{-1}\right) \cdots\left(C_{ \pm} \gamma^{\bar{\imath}_{k}} C_{ \pm}^{-1}\right) C_{ \pm} \mid \Omega>\right) \\
& =( \pm 1)^{k} \gamma^{i_{1}} \cdots \gamma^{i_{k}} C_{ \pm} \mid \Omega>.
\end{aligned}
$$

It follows from (73) that

$$
C_{ \pm}\left|\Omega>=\gamma^{\overline{1}} \cdots \gamma^{\bar{n}}\right| \Omega>
$$

and so

$$
\begin{aligned}
C_{ \pm}\left(\gamma^{\bar{\imath}_{1}} \cdots \gamma^{\bar{\imath}_{k}} \mid \Omega>\right) & =( \pm 1)^{k} \gamma^{i_{1}} \cdots \gamma^{i_{k}} \gamma^{\overline{1}} \cdots \gamma^{\bar{n}^{n}} \mid \Omega> \\
& =( \pm 1)^{k} \frac{(-1)^{k(k-1) / 2}}{(n-k) !} \epsilon^{i_{1} \cdots i_{n}} \gamma^{\bar{\imath}_{k+1}} \cdots \gamma^{\bar{\imath}_{n}} \mid \Omega> \\
& =\frac{(-1)^{k(k \mp 1) / 2}}{(n-k) !} \epsilon^{i_{1} \cdots i_{n}} \gamma^{\bar{\imath}_{k+1}} \cdots \gamma^{\bar{\imath}_{n}} \mid \Omega>
\end{aligned}
$$

Allowing for the fact that charge conjugation complex conjugates the coefficients, the final expression for the charge conjugate of a spinor,

$$
\Psi=\left(\psi+\psi_{\bar{\imath}} \gamma^{\bar{\imath}}+\cdots+\frac{1}{k !} \psi_{\bar{\imath}_{1} \cdots \bar{\imath}_{k}} \gamma^{\bar{\imath}_{1}} \cdots \gamma^{\bar{\imath}_{k}}+\cdots+\frac{1}{n !} \psi_{\bar{\imath}_{1} \cdots \bar{\imath}_{n}} \gamma^{\bar{\imath}_{1}} \cdots \gamma^{\bar{\imath}_{n}}\right) \mid \Omega>
$$

is

$$
\Psi_{c}:=\mathcal{C}(\Psi)=\sum_{k=0}^{n} \frac{(-1)^{k(k \mp 1) / 2}}{k !(n-k) !} \epsilon^{i_{1} \cdots i_{n}}\left(\psi_{\bar{\imath}_{1} \cdots \bar{\imath}_{k}}\right)^{*} \gamma^{\bar{\imath}_{k+1}} \cdots \gamma^{\bar{\imath}_{n}} \mid \Omega>.
$$

Charge conjugation maps between bundles:

$$
\mathcal{C}: S_{q}\left(\mathbb{C P}^{n}\right) \rightarrow S_{-q+n+1}\left(\mathbb{C P}^{n}\right) .
$$

In the fuzzy case we can still use (78) as the definition of charge conjugation acting on a spinor but the co-efficients $\psi_{\bar{\imath}_{1} \ldots \bar{\imath}_{k}}$ are replaced by linear combinations of the Fock space operators

$$
A_{\alpha_{1}}^{\dagger} \cdots A_{\alpha_{L+q-k}}^{\dagger}|0><0| A^{\beta_{1}} \cdots A^{\beta_{L}} a_{\alpha_{L+q-k+1}}^{\left[\imath_{1}\right.} \cdots a_{\alpha_{L+q}}^{\left.\imath_{k}\right]}
$$

and $\epsilon^{\imath_{1} \cdots \imath_{n}}\left(\psi_{\bar{\imath}_{1} \cdots \bar{\imath}_{k}}\right)^{*}$ by linear combinations of

$$
\begin{aligned}
\epsilon^{\imath_{i} \cdots \imath_{n}} & \left(A_{\alpha_{1}}^{\dagger} \cdots A_{\alpha_{L+q-k}}^{\dagger}|0><0| A^{\beta_{1}} \cdots A^{\beta_{L}} a_{\alpha_{L+q-k+1}}^{\imath_{1}} \cdots a_{\alpha_{L+q}}^{\imath_{k}}\right)^{\dagger} \\
& =\epsilon^{\imath_{i} \cdots \imath_{n}}\left(a^{\dagger}\right)_{\imath_{1}}^{\alpha_{L+q-k+1}} \cdots\left(a^{\dagger}\right)_{\imath_{k}}^{\alpha_{L+q}} A_{\beta_{1}}^{\dagger} \cdots A_{\beta_{L}}^{\dagger}|0><0| A^{\alpha_{1}} \cdots A^{\alpha_{L+q-k}} .
\end{aligned}
$$




\section{Conclusions}

We have extended the previous construction of noncommutative equivariant vector bundles over fuzzy $\mathbb{C P}^{n}$ in [24] to noncommutative spin and $\operatorname{spin}^{\mathrm{c}}$ bundles $\mathcal{S}_{q}\left(\mathbb{C P}^{n}\right)$ (the former only exist for odd $n$ ), where $q$ is a power of the tautological line bundle $\mathbf{L}$ in (32) — physically it represents a background monopole to which the spinor couples with charge $q$. In the continuum a general spinor (43) can be decomposed into a sum of irreducible representations of the $S U(n)$ part of the holonomy group $U(n)$. Only the totally anti-symmetric irreps of $S U(n)$, labelled by an index $k=0, \cdots, n$, occur. The trivial representation appears twice, for $k=0$ and $k=n$, with different $U(1)$ charges. Each of these $U(n)$ irreps itself has a harmonic expansion in terms of irreducible representations of the isometry group $S U(n+1)$ and these determine the eigenvalues (50) and degeneracies (51) of the Dirac operator. Although the different $S U(n)$ irreps have different $U(1)$ charges the total $U(1)$ charge on a spinor is the same for all components, because the $\gamma$-matrices in (43) themselves carry charge. The total charge is $Q=q-\frac{n+1}{2}$ where the term $-\frac{n+1}{2}$ is gravitational, i.e. it comes from the $U(1)$ part of the Riemann curvature.

In the fuzzy case a general spinor has the form given in (63) and (64). The components are non-square matrices representing operators on a Fock space which is generated by $n(n+1)$ creation and annihilation operators, $\left(a^{\dagger}\right)_{i}^{\alpha}$ and $a_{\alpha}^{\imath}$, carrying the fundamental representation of $s u(n+1) \times u(n)$, with $U(1)$ charge $+1 / n$ and $-1 / n$ respectively. For the $k=n$ and $k=0$ components of the spinor (the $s u(n)$ singlets) only composite operators, $A_{\alpha}^{\dagger}$ and $A^{\alpha}$ in (10) and (11), are required.

One of the more elegant ingredients in the construction is the appearance of a universal Dirac operator (49), with $K_{\imath}$ and $K_{\bar{\imath}}$ given in (33), which generalises the $\mathbb{C P}^{1}$ case developed in [17]. This universal Dirac operator has the same form on all the bundles $\mathcal{S}_{q}\left(\mathbb{C P}^{n}\right)$, the construction automatically takes care of the different connections for the the different bundles.

Having constructed fuzzy spinors in a consistent manner we hope in the future to investigate the possibility of coupling them to other fuzzy fields, through Yukawa and gauge couplings, with a view to performing numerical simulations with a finite number of degrees of freedom, as suggested in [4]. 


\section{A Eigenspinors of the Dirac operator}

In this appendix we determine the eigenspinors of the operator (49). The continuum $S U(n+1)$ representation theory of exact, co-exact and harmonic $\mathbf{L}^{\frac{n+1}{2}}$-valued $(0, k)$-forms on $\mathbb{C P}^{n}$, for $n$ odd, is known [30, 31, 32]. This imposes very strong restrictions on the structure of modules corresponding to these forms. The modules which are described here show that our choice of Dirac operator gives the correct spectrum, with just a cut-off at the noncommutative level given by $L$, for the continuum cases known to us: spin structure for odd $n[30,31,32,33]$ and $\operatorname{spin}^{c}$ structures for $\mathbb{C P}^{2}[34,35]$.

The modules discussed in the text, associated with the line bundles $\mathbf{L}^{q}$, are carrier spaces for $s u(n+1)$ representations and decompose into irreducibles $\operatorname{as}^{4}$

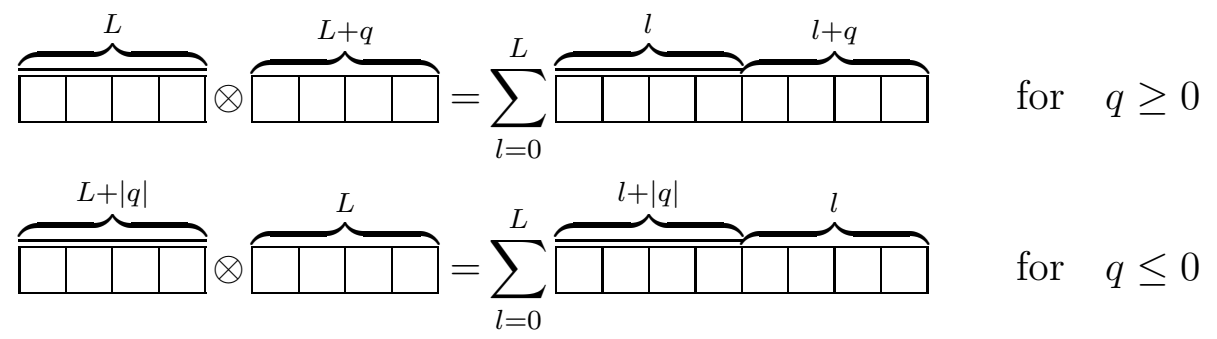

respectively. We shall prefer to use a convention consistent with all values of $q$. Therefore we shall always write modules corresponding to $\mathbf{L}^{q}$ as

$$
\mathcal{F}_{L+q} \otimes \mathcal{F}_{L}^{*}
$$

and write their representation content as

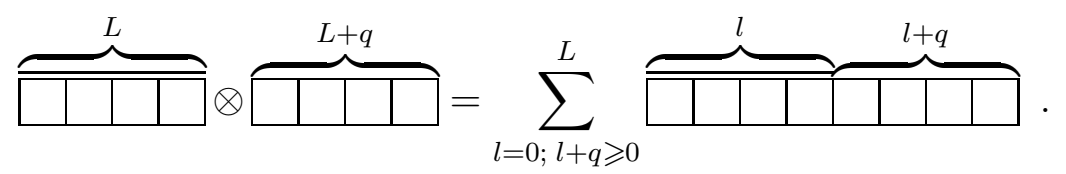

Here the sum over $l$ is from 0 to $L$ but with the constraint that $l+q \geqslant 0$. For $q<0$ this differs from (81) above only by a change in the cut-off $L$.

We can now write down the space of $\overline{\mathcal{D}}$-exact noncommutative $(0,1)$-forms as the left module

$$
\overline{\mathcal{D}}\left(\mathcal{F}_{L+q} \otimes \mathcal{F}_{L}^{*} \otimes|\Omega\rangle\right) \subset \mathcal{F}_{L+q-1} \otimes \mathcal{F}_{L, \bar{\imath}}^{*} \otimes \gamma^{\bar{\imath}}|\Omega\rangle .
$$

\footnotetext{
${ }^{4}$ For convenience we represent anti-fundamental representations on young tableaux by

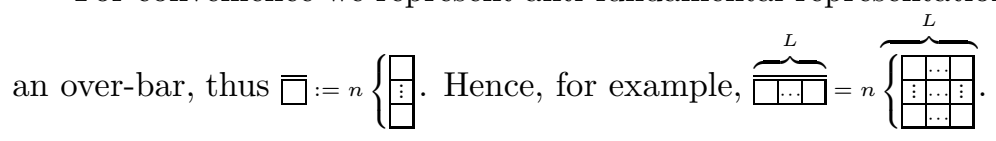


This space has basis elements

$$
\mid\{\boldsymbol{\alpha}\rangle\left\langle\boldsymbol{\beta}\left|a_{\left.\alpha_{L+q}\right\}}^{\imath} \otimes \gamma^{\bar{\imath}}\right| \Omega\right\rangle
$$

with $\alpha_{l}, l=1 \ldots L+q$, symmetrised and decomposes as

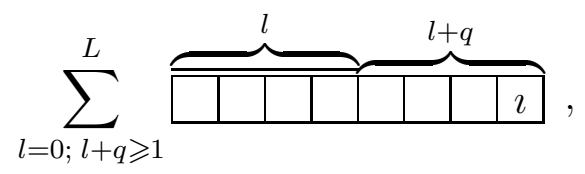

where $\square$ represents the oscillator $a_{\alpha}^{\imath}$ acting on the right as opposed to $A_{\alpha}^{\dagger}$ acting on the left. A natural generalisation presents itself: we expect the space of all noncommutative $(0,1)$-forms $\Lambda^{0,1}\left(\mathbb{C} \mathbb{P}^{n}, q\right)$ to be given by the module

$$
\Lambda^{0,1}\left(\mathbb{C P}^{n}, q\right):=\mathcal{F}_{L+q-1} \otimes \mathcal{F}_{L, \bar{\imath}}^{*} \otimes \gamma^{\bar{\imath}}|\Omega\rangle=\operatorname{span}\left(|\boldsymbol{\alpha}\rangle\left\langle\boldsymbol{\beta}\left|a_{\alpha_{L+q}}^{\imath} \otimes \gamma^{\bar{\imath}}\right| \Omega\right\rangle\right)
$$

Its representation theory content is

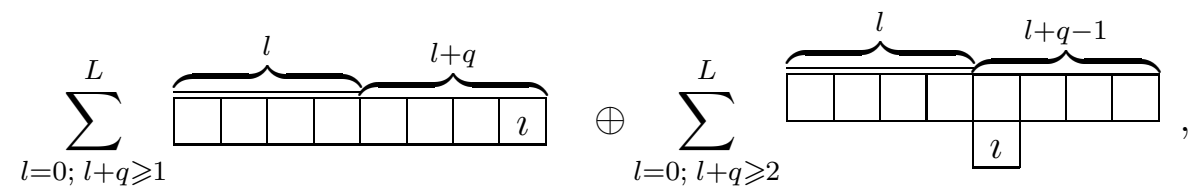

where the latter term corresponds to the space of harmonic and $\overline{\mathcal{D}}^{\dagger}$-exact $(0,1)$-forms, which we shall refer to as $\overline{\mathcal{D}}$-nonexact forms ${ }^{5}$. It is spanned by

$$
A_{\left[\alpha_{L+q-1}\right.}^{\dagger}|\boldsymbol{\alpha}\rangle\left\langle\boldsymbol{\beta}\left|a_{\left.\alpha_{L+q}\right]}^{\imath} \otimes \gamma^{\bar{\imath}}\right| \Omega\right\rangle
$$

and where in the usual interpretation of Young tableaux, $\alpha_{1} \cdots \alpha_{L+q-1}$ are symmetrised first and then $\alpha_{L+q-1}$ and $\alpha_{L+q}$ are anti-symmetrised.

We can write this sum as a tensor product

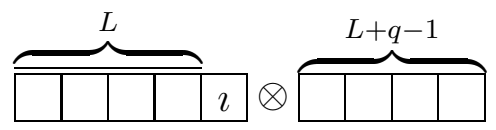

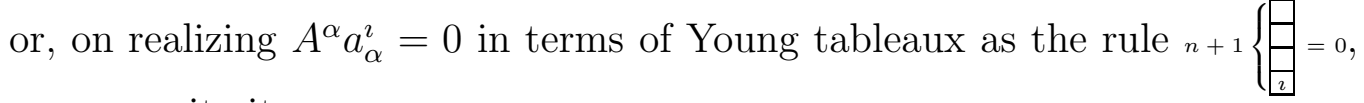
we can write it as

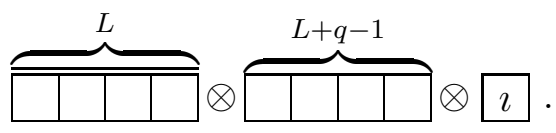

\footnotetext{
${ }^{5}$ We shall see presently that harmonic forms (which are zero modes of $\not D$ ) exist only for $k=0$ or $n$.
} 
We can continue in this fashion to write down all modules. Acting with $\overline{\mathcal{D}}$ on the space of nonexact $(0,1)$-forms, we obtain exact $(0,2)$-forms. Then, as before, we can write the bases of all noncommutative $(0,2)$-forms, exact and non-exact, as

$$
\Lambda^{0,2}\left(\mathbb{C P}^{n}, q\right):=\mathcal{F}_{L+q-2} \otimes \mathcal{F}_{L, \bar{\nu}_{1} \bar{\tau}_{2}}^{*} \otimes \gamma^{\bar{\tau}_{1}} \gamma^{\bar{\tau}_{2}}|\Omega\rangle,
$$

as

$$
\begin{aligned}
& A_{\alpha_{1}}^{\dagger} \cdots A_{\alpha_{L+q-2}}^{\dagger}|0\rangle\left\langle 0\left|A^{\beta_{1}} \cdots A^{\beta_{L}} a_{\alpha_{L+q-1}}^{\imath_{1}} a_{\alpha_{L+q}}^{\imath_{2}} \otimes \gamma^{\overline{1}_{1}} \gamma^{\bar{z}_{2}}\right| \Omega\right\rangle \quad \text { all } \\
& A_{\left\{\alpha_{1}\right.}^{\dagger} \cdots A_{\alpha_{L+q-2}}^{\dagger}|0\rangle\left\langle 0\left|A^{\beta_{1}} \cdots A^{\beta_{L}} a_{\left.\alpha_{L+q-1}\right\}}^{a_{1}} a_{\alpha_{L+q}}^{l_{2}} \otimes \gamma^{\overline{1}_{1}} \gamma^{\bar{\tau}_{2}}\right| \Omega\right\rangle \quad \text { exact } \\
& A_{\alpha_{1}}^{\dagger} \cdots A_{\left[\alpha_{L+q-2}\right.}^{\dagger}|0\rangle\left\langle 0\left|A^{\beta_{1}} \cdots A^{\beta_{L}} a_{\alpha_{L+q-1}}^{l_{1}} a_{\left.\alpha_{L+q}\right]}^{\imath_{2}} \otimes \gamma^{\bar{\nu}_{1}} \gamma^{\bar{\tau}_{2}}\right| \Omega\right\rangle \quad \text { non-exact }
\end{aligned}
$$

and their representation content is

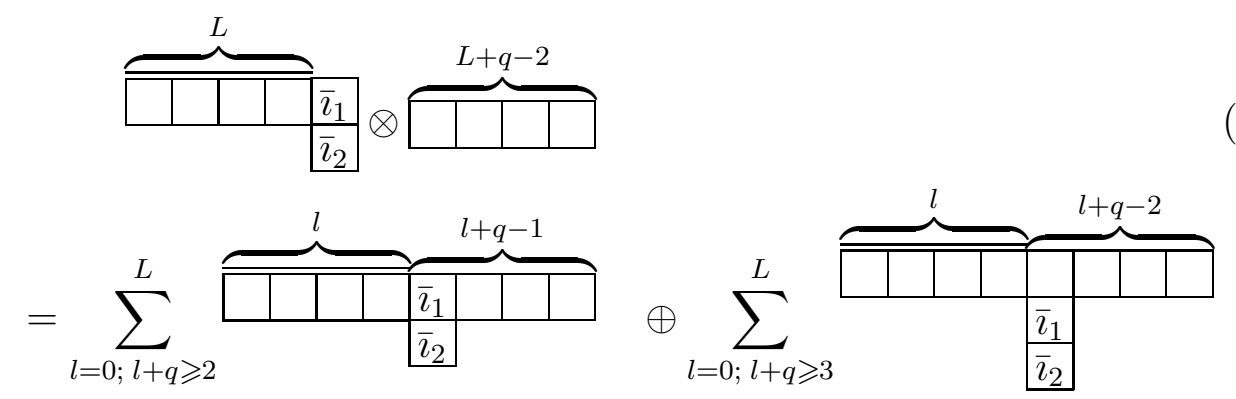

where the first sum on the right hand side represents exact forms and the second non-exact forms.

Repeating the procedure, we can write down the modules describing noncommutative $\mathbf{L}^{q}$-valued $(0, k)$-forms as

$$
\Lambda^{0, k}\left(\mathbb{C P}^{n}, q\right):=\mathcal{F}_{L+q-k} \otimes \mathcal{F}_{L, \bar{z}_{1} \cdots \bar{z}_{k}}^{*} \otimes \gamma^{\overline{1}_{1}} \cdots \gamma^{\bar{\tau}_{k}}|\Omega\rangle .
$$

For $k=1, \ldots, n-1$, the representation content breaks into exact and nonexact forms as
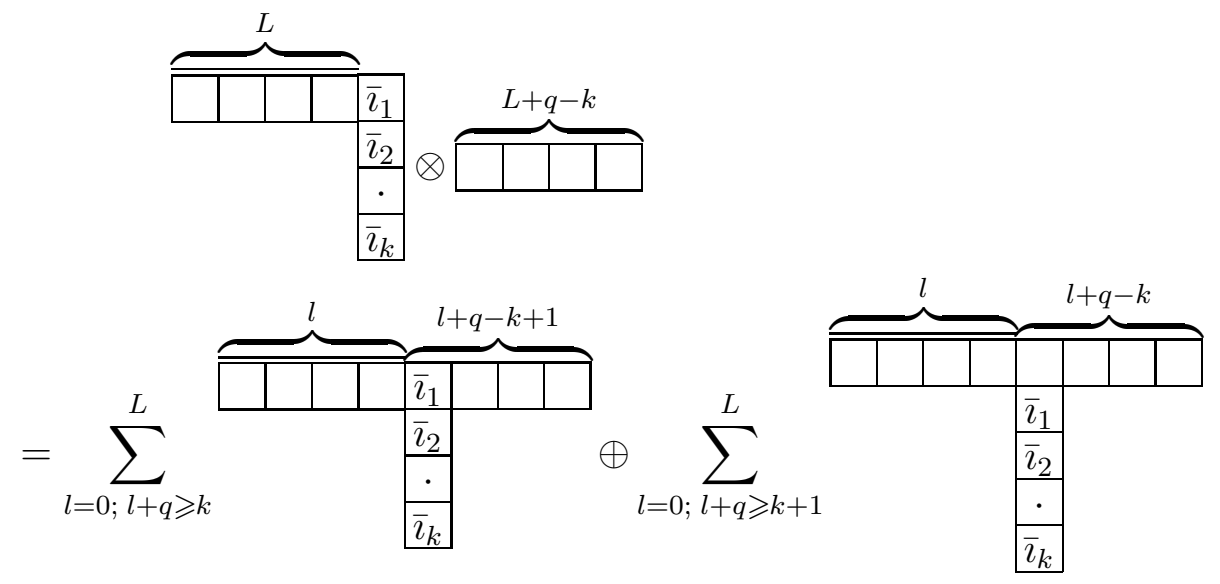
respectively. The right-hand side of equation (96) is valid for $k=1 \ldots n-1$.

For $k=0$ the representation content is that of (83). This contains zero modes of the Dirac operator for $q \leq 0$, as given in (60), namely

$$
\left|\boldsymbol{\mu}_{L}\right\rangle\left\langle\boldsymbol{\mu}_{L} \boldsymbol{\alpha}_{|q|}|\otimes| \Omega\right\rangle, \quad \overbrace{\overbrace{\square|l|}^{|q|},}^{|q|}
$$

which are annihilated by $\not D$ since $\hat{K}_{\bar{\imath}} \mathbf{1}=0$.

For $k=n$ the decomposition of $\mathcal{F}_{L+q-n} \otimes \mathcal{F}_{L+1}^{*}$ is

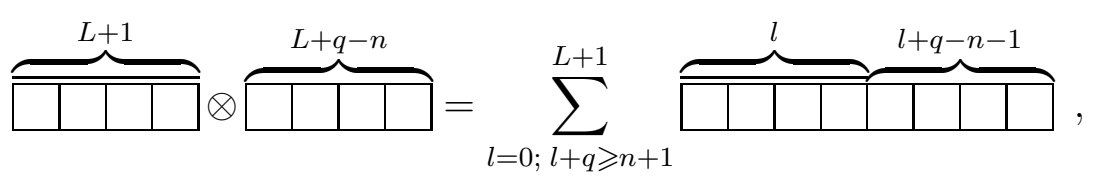

which contains zero modes, as given in (61), when $q \geqslant n+1$

$$
\left|\boldsymbol{\alpha}_{q-n-1} \boldsymbol{\mu}_{L+1}\right\rangle\left\langle\boldsymbol{\mu}_{L+1}\left|\otimes \gamma^{\overline{1}} \cdots \gamma^{\bar{n}}\right| \Omega\right\rangle, \quad \overbrace{\square \square}^{q-n-1} .
$$

They are zero modes of the Dirac operator since $\hat{K}_{\imath} \mathbf{1}=0$. There are no zero modes for $0<q<n+1$.

Therefore the tensor product (96) contains just the representations which are carried by eigenoperators of $\not D^{2}$ that have non-zero eigenvalues.

For completeness we give the dimension of the irreducible representations given above:

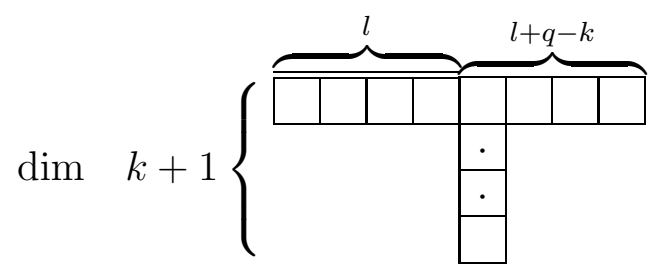

$$
\begin{aligned}
& =\frac{(l+n) !(l+q-k-1+n) !(2 l+q-k+n)}{l ! k ! n !(l+q-k-1) !(n-k-1) !(l+n-k)(l+q)} \text {. }
\end{aligned}
$$

Therefore $\boldsymbol{\Lambda}^{0, k}\left(\mathbb{C P}^{n}, q\right)$ has dimension

$$
\operatorname{dim}\left(\Lambda^{0, k}\left(\mathbb{C P}^{n}, q\right)\right)=\frac{(L+n+1) !}{L ! k !(n-k) !(L+n-k+1)} \frac{(L+q-k+n) !}{(L+q-k) ! n !}
$$

We will now write eigenspinors of the Dirac operator. We have seen that the space of $\overline{\mathcal{D}}$-nonexact $(0, k)$-forms, $k=0, \ldots, n-1$ breaks up into 
irreducible representations

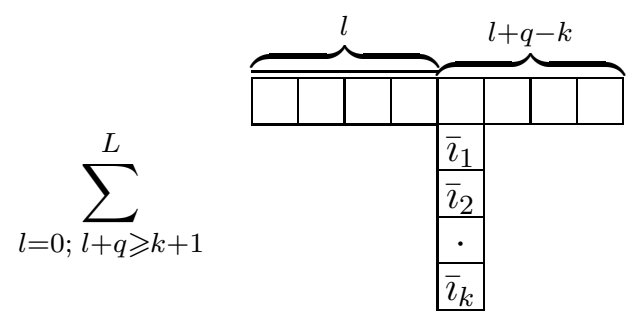

of $s u(n+1)$. The $l^{\text {th }}$ mode is spanned by the elements

$$
\begin{aligned}
& \boldsymbol{\psi}_{\gamma_{l+q}}^{k} \boldsymbol{\delta}_{l}=\mathcal{P}_{\boldsymbol{\gamma}_{l+q} \boldsymbol{\beta}_{l}} \boldsymbol{\delta}_{l} \boldsymbol{\alpha}_{l+q} A_{\alpha_{l+q}}^{\dagger} \cdots A_{\alpha_{k+2}}^{\dagger} A_{\left[\alpha_{k+1}\right.}^{\dagger}\left|\boldsymbol{\mu}_{L-l}\right\rangle\left\langle\boldsymbol{\mu}_{L-l} \boldsymbol{\beta}_{l}\right| a_{\alpha_{1}}^{\imath_{1}} \cdots a_{\left.\alpha_{k}\right]}^{\imath_{k}} \\
& \otimes \gamma^{\bar{\imath}_{1}} \cdots \gamma^{\bar{c}_{k}}|\Omega\rangle \\
& =\mathcal{P}_{\boldsymbol{\gamma}_{l+q} \boldsymbol{\beta}_{l}} \boldsymbol{\delta}_{l} \boldsymbol{\alpha}_{l+q} A_{\left[\alpha_{k+1}\right.}^{\dagger}\left|\alpha_{k+2} \cdots \alpha_{l+q} \boldsymbol{\mu}_{L-l}\right\rangle\left\langle\boldsymbol{\mu}_{L-l} \boldsymbol{\beta}_{l}\right| a_{\alpha_{1}}^{\imath_{1}} \cdots a_{\left.\alpha_{k}\right]}^{\imath_{k}} \\
& \otimes \gamma^{\bar{\imath}_{1}} \cdots \gamma^{\bar{\imath}_{k}}|\Omega\rangle
\end{aligned}
$$

where the projector $\mathcal{P}$ removes all $\boldsymbol{\alpha} \leftrightarrow \boldsymbol{\beta}$ contractions and anti-symmetrisation is over the indices $\alpha_{1}, \ldots, \alpha_{k+1}$. In appendix B we show that $\boldsymbol{\psi}_{\boldsymbol{\alpha}_{l+q}}^{k} \boldsymbol{\beta}_{l}$ is an eigenoperator of $\not D^{2}=\overline{\mathcal{D}} \overline{\mathcal{D}}^{\dagger}+\overline{\mathcal{D}}^{\dagger} \overline{\mathcal{D}}=\gamma^{\bar{\imath}} \gamma^{\jmath} \hat{K}_{\bar{\imath}} \hat{K}_{j}+\gamma^{\jmath} \gamma^{\bar{\imath}} \hat{K}_{j} \hat{K}_{\bar{\imath}}$

$$
\not D^{2} \boldsymbol{\psi}_{\boldsymbol{\alpha}_{l+q}}^{k} \boldsymbol{\beta}_{l}=\lambda^{2} \boldsymbol{\psi}_{\boldsymbol{\alpha}_{l+q}}^{k} \boldsymbol{\beta}_{l}
$$

with non-zero eigenvalues

$$
\lambda^{2}=(l+q)(l-k+n), \quad l=0 \ldots L \text { subject to } l+q \geqslant k+1,
$$

with $k=0, \ldots, n-1$. Eigenspinors $\Psi^{ \pm}$are therefore given by

$$
\Psi_{\boldsymbol{\alpha}_{l+q}}^{ \pm, k \quad \boldsymbol{\beta}_{l}}:=\boldsymbol{\psi}_{\boldsymbol{\alpha}_{l+q}}^{k \quad \boldsymbol{\beta}_{l}} \pm \frac{1}{\lambda} \not D \psi_{\boldsymbol{\alpha}_{l+q}}^{k \quad \boldsymbol{\beta}_{l}}
$$

with eigenvalues $\pm \lambda$ and degeneracies given by the dimension (100) of the relevant $s u(n+1)$ irreducible representations.

These values agree, up to the cut-off at $L$, with the available continuum spectrum and degeneracies given for spin structure on $\mathbb{C P}^{n}[30,31,32,33]$ and $\operatorname{spin}^{\mathrm{c}}$ structures on $\mathbb{C P}^{2}[34,35]$. The number of, and conditions for, zeros modes of the Dirac operator, given in (97) and (99) also agrees with the continuum result [28].

Note that the space of spinors for a given $L$ and $q$ is a linear combination of left modules corresponding to different algebras, $\mathcal{A}_{L+q-k}, k=0, \ldots, n$. 


\section{B Eigenvalues of the Dirac operator}

In this appendix we shall calculate the eigenvalues of the square of the Dirac operator. Consider an $l$-th mode nonexact $(0, k)$-form basis element $\boldsymbol{\psi}_{\gamma_{l+g}}^{k} \boldsymbol{\delta}_{l}$ described in (103), with $l+q+k \geq 1$ and $0 \leq k \leq n-1$. Observing that $\hat{K}_{i}\left(\boldsymbol{\psi}_{\gamma_{l+q}}^{k} \boldsymbol{\delta}_{l}\right)=0$ we find that acting with $\not D^{2}$ gives

$$
\begin{aligned}
\not D^{2} \boldsymbol{\psi}_{\gamma_{l+q}}^{k} \boldsymbol{\delta}_{l} & =\overline{\mathcal{D}}^{\dagger} \overline{\mathcal{D}} \boldsymbol{\psi}_{\gamma_{l+q}}^{k} \boldsymbol{\delta}_{l} \\
& =\hat{K}_{\imath} \hat{K}_{\bar{\jmath}} \gamma^{\imath} \gamma^{\bar{\jmath}} \boldsymbol{\psi}_{\gamma_{l+q}^{k}}^{k} \boldsymbol{\delta}_{l} \\
& =\hat{K}_{\imath} \hat{K}_{\bar{\imath}} \boldsymbol{\psi}_{\gamma_{l+q}}^{k} \boldsymbol{\delta}_{l}-\hat{K}_{\imath} \hat{K}_{\bar{\jmath}} \gamma^{\bar{j}} \gamma^{\imath} \boldsymbol{\psi}_{\gamma_{l+q}}^{k} \boldsymbol{\delta}_{l}
\end{aligned}
$$

Taking each term individually:

$$
\begin{aligned}
& \hat{K}_{\imath} \hat{K}_{\bar{\imath}} \boldsymbol{\psi}_{\gamma_{l+q}}^{k} \boldsymbol{\delta}_{l}=\mathcal{P}_{\boldsymbol{\gamma}_{l+q} \boldsymbol{\beta}_{l}} \boldsymbol{\delta}_{l} \boldsymbol{\alpha}_{l+q} A_{\eta_{1}}^{\dagger} A^{\eta_{2}} A_{\left[\alpha_{k+1}\right.}^{\dagger}\left|\alpha_{k+2} \cdots \alpha_{l+q} \boldsymbol{\mu}_{L-l}\right\rangle \\
& \left\langle\boldsymbol{\mu}_{L-l} \boldsymbol{\beta}\left|a_{\alpha_{1}}^{\imath_{1}} \cdots a_{\left.\alpha_{k}\right]}^{\imath_{k}} a_{\eta_{2}}^{\imath}\left(a^{\dagger}\right)_{\imath}^{\eta_{1}} \otimes \gamma^{\overline{1}_{1}} \cdots \gamma^{\bar{i}_{k}}\right| \Omega\right\rangle \\
& =\hat{K}_{\imath} \hat{K}_{\bar{\imath}}\left(\mathcal{P}_{\gamma_{l+q} \boldsymbol{\beta}_{l}}{ }^{\boldsymbol{\delta}_{l} \boldsymbol{\alpha}_{l+q}} A_{\left[\alpha_{k+1}\right.}^{\dagger}\left|\alpha_{k+2} \cdots \alpha_{l+q} \boldsymbol{\mu}_{L-l}\right\rangle\left\langle\boldsymbol{\mu}_{L-l} \boldsymbol{\beta}\right|\right) a_{\alpha_{1}}^{\imath_{1}} \cdots a_{\left.\alpha_{k}\right]}^{\imath_{k}} \\
& \otimes \gamma^{\bar{i}_{1}} \cdots \gamma^{\bar{i}_{k}}|\Omega\rangle \\
& +\sum_{j=1}^{k} \mathcal{P}_{\gamma_{l+q} \boldsymbol{\beta}_{l}} \boldsymbol{\delta}_{l} \boldsymbol{\alpha}_{l+q} A_{\alpha_{j}}^{\dagger} A^{\eta_{2}} A_{\left[\alpha_{k+1}\right.}^{\dagger}\left|\alpha_{k+2} \cdots \alpha_{l+q} \boldsymbol{\mu}_{L-l}\right\rangle \\
& \left\langle\boldsymbol{\mu}_{L-l} \boldsymbol{\beta}\left|a_{\alpha_{1}}^{\imath_{1}} \cdots a_{\alpha_{j-1}}^{\imath_{j-1}} a_{\eta_{2}}^{\imath_{j}} a_{\alpha_{j+1}}^{\imath_{j+1}} \cdots a_{\left.\alpha_{k}\right]}^{\imath_{k}} \otimes \gamma^{\bar{\imath}_{1}} \cdots \gamma^{\bar{\imath}_{k}}\right| \Omega\right\rangle \\
& =((l+q-k)(l+n)-k) \boldsymbol{\psi}_{\gamma_{l+q}}^{k} \boldsymbol{\delta}_{l}
\end{aligned}
$$

where we have used (14), (38) and antisymmetry of $\alpha_{1} \cdots \alpha_{k+1}$.

We now see that

$$
\begin{aligned}
& \hat{K}_{\imath} \hat{K}_{\bar{\jmath}} \gamma^{\bar{\gamma}} \gamma^{\imath} \boldsymbol{\psi}_{\boldsymbol{\gamma}_{l+q}}^{k} \boldsymbol{\delta}_{l}=\sum_{j=1}^{k}(-1)^{j-1} \hat{K}_{\imath_{j}} \hat{K}_{\bar{\imath}} \mathcal{P}_{\boldsymbol{\gamma}_{l+q} \boldsymbol{\beta}_{l}} \boldsymbol{\delta}_{l} \boldsymbol{\alpha}_{l+q} A_{\left[\alpha_{k+1}\right.}^{\dagger}\left|\alpha_{k+2} \cdots \alpha_{l+q} \boldsymbol{\mu}_{L-l}\right\rangle \\
& \left\langle\boldsymbol{\mu}_{L-l} \boldsymbol{\beta}\left|a_{\alpha_{1}}^{\imath_{1}} \cdots a_{\left.\alpha_{k}\right]}^{\imath_{k}} \otimes \gamma^{\bar{\imath}} \gamma^{\bar{i}_{1}} \cdots \gamma^{\bar{i}_{j-1}} \gamma^{\bar{i}_{j+1}} \cdots \gamma^{\bar{i}_{k}}\right| \Omega\right\rangle \\
& =k \mathcal{P}_{\gamma_{l+q} \boldsymbol{\beta}_{l}} \boldsymbol{\delta}_{l} \boldsymbol{\alpha}_{l+q} A_{\eta_{1}}^{\dagger} A^{\eta_{2}} A_{\left[\alpha_{k+1}\right.}^{\dagger}\left|\alpha_{k+2} \cdots \alpha_{l+q} \boldsymbol{\mu}_{L-l}\right\rangle \\
& \left\langle\boldsymbol{\mu}_{L-l} \boldsymbol{\beta}\left|a_{\alpha_{1}}^{\imath_{1}} \cdots a_{\left.\alpha_{k}\right]}^{\imath_{k}} a_{\eta_{2}}^{\imath_{2}}\left(a^{\dagger}\right)_{\imath_{1}}^{\eta_{1}} \otimes \gamma^{\bar{i}} \gamma^{\bar{\tau}_{2}} \cdots \gamma^{\bar{\imath}_{k}}\right| \Omega\right\rangle \\
& =-k \mathcal{P}_{\gamma_{l+q} \boldsymbol{\beta}_{l}} \boldsymbol{\delta}_{l} \boldsymbol{\alpha}_{l+q}\left(\delta_{\eta_{1}}^{\eta_{2}}-A^{\eta_{2}} A_{\eta_{1}}^{\dagger}\right) A_{\left[\alpha_{k+1}\right.}^{\dagger}\left|\alpha_{k+2} \cdots \alpha_{l+q} \boldsymbol{\mu}_{L-l}\right\rangle \\
& \left\langle\boldsymbol{\mu}_{L-l} \boldsymbol{\beta}\left|a_{\alpha_{1}}^{\imath_{1}} \cdots a_{\left.\alpha_{k}\right]}^{\imath_{k}}\left(\left(a^{\dagger}\right)_{\imath_{1}}^{\eta_{1}} a_{\eta_{2}}^{\imath}+\delta_{l_{1}}^{\imath} \delta_{\eta_{2}}^{\eta_{1}}\right) \otimes \gamma^{\bar{\tau}} \gamma^{\bar{\tau}_{2}} \cdots \gamma^{\bar{\tau}_{k}}\right| \Omega\right\rangle \\
& =-k[(n+1)-(n+1+L+q-k)] \boldsymbol{\psi}_{\gamma_{l+q}}^{k} \boldsymbol{\delta}_{l} \\
& -k \mathcal{P}_{\gamma_{l+q} \boldsymbol{\beta}_{l}} \boldsymbol{\delta}_{l} \boldsymbol{\alpha}_{l+q} A_{\left[\alpha_{k+1}\right.}^{\dagger}\left|\alpha_{k+2} \cdots \alpha_{l+q} \boldsymbol{\mu}_{L-l}\right\rangle \\
& \left\langle\boldsymbol{\mu}_{L-l} \boldsymbol{\beta}\left|a_{\alpha_{1}}^{\imath_{1}} \cdots a_{\left.\alpha_{k}\right]}^{\imath_{k}} \hat{J}_{l_{1}}{ }^{\imath} \otimes \gamma^{\bar{\imath}} \gamma^{\bar{\tau}_{2}} \cdots \gamma^{\bar{i}_{k}}\right| \Omega\right\rangle,
\end{aligned}
$$


where we have used the fact that one of the cross-terms vanishes, since

$$
\begin{aligned}
& -A^{\eta_{2}} A_{\eta_{1}}^{\dagger} A_{\left[\alpha_{k+1}\right.}^{\dagger}\left|\alpha_{k+2} \cdots \alpha_{l+q} \boldsymbol{\mu}_{L-l}\right\rangle\left\langle\boldsymbol{\mu}_{L-l} \boldsymbol{\beta}\right| a_{\alpha_{1}}^{\imath_{1}} \cdots a_{\left.\alpha_{k}\right]}^{\imath_{k}}\left(a^{\dagger}\right)_{l_{1}}^{\eta_{1}} a_{\eta_{2}}^{\imath} \\
= & -A^{\eta_{2}} A_{\eta_{1}}^{\dagger} A_{\left[\alpha_{k+1}\right.}^{\dagger}\left|\alpha_{k+2} \cdots \alpha_{l+q} \boldsymbol{\mu}_{L-l}\right\rangle\left\langle\boldsymbol{\mu}_{L-l} \boldsymbol{\beta}\right|\left(a^{\dagger}\right)_{\imath_{1}}^{\eta_{1}} a_{\alpha_{1}}^{\imath_{1}} \cdots a_{\left.\alpha_{k}\right]}^{\imath_{k}} a_{\eta_{2}}^{\imath} \\
= & -A^{\eta_{2}} A_{\eta_{1}}^{\dagger} A_{\left[\alpha_{k+1}\right.}^{\dagger}\left|\alpha_{k+2} \cdots \alpha_{l+q} \boldsymbol{\mu}_{L-l}\right\rangle\left\langle\boldsymbol{\mu}_{L-l} \boldsymbol{\beta}\right| \hat{J}_{\alpha_{1}}^{\eta_{1}} a_{\alpha_{2}}^{\imath_{2}} \cdots a_{\left.\alpha_{k}\right]}^{\iota_{k}} a_{\eta_{2}}^{\imath}
\end{aligned}
$$

and (21) shows that $\mathcal{P}_{\boldsymbol{\gamma} \boldsymbol{\beta}}{ }^{\boldsymbol{\alpha} \boldsymbol{\alpha}} A_{\eta_{1}}^{\dagger} A_{\left[\alpha_{k+1}\right.}^{\dagger}\left|\boldsymbol{\alpha} \boldsymbol{\mu}_{L-l}\right\rangle\left\langle\boldsymbol{\mu}_{L-l} \boldsymbol{\beta}\right| \hat{J}^{\eta_{1}}{ }_{\left.\alpha_{1}\right]}=0$ since $\mathcal{P}_{\boldsymbol{\gamma} \boldsymbol{\beta}}{ }^{\boldsymbol{\alpha} \boldsymbol{\alpha}}$ removes all contractions between upper and lower indices. Finally commuting $\hat{J}_{\imath_{1}}{ }^{\imath}$ in the last term on the right hand side of (109) through the $a_{\alpha_{j}}^{\imath_{j}}$ to use (19) and noticing the commutator terms give a factor of $n-(k-1)$ we find

$$
\begin{aligned}
\hat{K}_{\imath} \hat{K}_{\bar{\jmath}} \gamma^{\bar{\jmath}} \gamma^{\imath} \boldsymbol{\psi}_{\gamma_{l+q}}^{k} \boldsymbol{\delta}_{l} & =-k[(k-L-q)+L+n-(k-1)] \boldsymbol{\psi}_{\gamma_{l+q}}^{k} \boldsymbol{\delta}_{l} \\
& =-k(n-q+1) \boldsymbol{\psi}_{\gamma_{l+q}}^{k} \boldsymbol{\delta}_{l}
\end{aligned}
$$

Hence

$$
\not D^{2} \boldsymbol{\psi}_{\gamma_{l+q}}^{k} \boldsymbol{\delta}_{l}=\lambda^{2} \boldsymbol{\psi}_{\gamma_{l+q}}^{k} \boldsymbol{\delta}_{l}
$$

where $\lambda^{2}=(l+q)(l-k+n)$, with $l+q+k \geq 1$ and $0 \leq k \leq n-1$. As far as we know the $\operatorname{spin}^{c}$ spectrum presented here for $n>2$ is new.

\section{References}

[1] J. Madore, An introduction to noncommutative differential geometry and physical applications, Lond. Math. Soc. Lect. Note Ser. 257 (2000) $1-371$.

[2] N. Seiberg and E. Witten, String theory and noncommutative geometry, JHEP 09 (1999) 032, [hep-th/9908142].

[3] R. C. Myers, Dielectric-branes, JHEP 12 (1999) 022, [hep-th/9910053].

[4] H. Grosse, C. Klimcik, and P. Prešnajder, Towards finite quantum field theory in noncommutative geometry, Int. J. Theor. Phys. 35 (1996) 231-244, [hep-th/9505175].

[5] F. A. Berezin, General concept of quantization, Commun. Math. Phys. 40 (1975) 153-174.

[6] J. Hoppe, Quantum Theory of a Massless Relativistic Surface and a Two Dimensional Bound State Problem. PhD thesis, MIT, 1982. 
[7] J. Madore, The fuzzy sphere, Class. Quant. Grav. 9 (1992) 69-88.

[8] D. O'Connor, Field theory on low dimensional fuzzy spaces, Mod. Phys. Lett. A18 (2003) 2423-2430.

[9] D. O'Connor and C. Saemann, Fuzzy scalar field theory as a multitrace matrix model, JHEP 08 (2007) 066, [0706.2493].

[10] X. Martin, A matrix phase for the $\phi^{4}$ scalar field on the fuzzy sphere, JHEP 04 (2004) 077, [hep-th/0402230].

[11] F. Garcia Flores, D. O'Connor, and X. Martin, Simulating the scalar field on the fuzzy sphere, PoS LAT2005 (2006) 262, [hep-lat/0601012].

[12] M. Panero, Numerical simulations of a non-commutative theory: The scalar model on the fuzzy sphere, JHEP 05 (2007) 082, [hep-th/0608202].

[13] D. O'Connor and B. Ydri, Monte Carlo simulation of a NC gauge theory on the fuzzy sphere, JHEP 11 (2006) 016, [hep-lat/0606013].

[14] A. P. Balachandran, B. P. Dolan, J.-H. Lee, X. Martin, and D. O'Connor, Fuzzy complex projective spaces and their star-products, J. Geom. Phys. 43 (2002) 184-204, [hep-th/0107099].

[15] S. Murray and C. Saemann, Quantization of flag manifolds and their supersymmetric extensions, ATMP 12 (2008), no. 3 [hep-th/0611328].

[16] C. Saemann, Fuzzy toric geometries, JHEP 02 (2008) 111.

[17] H. Grosse and P. Prešnajder, The Dirac operator on the fuzzy sphere, Lett. Math. Phys. 33 (1995) 171-182.

[18] G. Alexanian, A. P. Balachandran, G. Immirzi, and B. Ydri, Fuzzy $C P^{2}$, J. Geom. Phys. 42 (2002) 28-53, [hep-th/0103023].

[19] A. P. Balachandran, G. Immirzi, J. Lee, and P. Prešnajder, Dirac operators on coset spaces, J. Math. Phys. 44 (2003) 4713-4735, [hep-th/0210297].

[20] E. Hawkins, Quantization of equivariant vector bundles, Commun. Math. Phys. 202 (1999) 517-546, [q-alg/9708030].

[21] E. Hawkins, Geometric quantization of vector bundles, Commun. Math. Phys. 215 (2000) 409-432, [math/9808116]. 
[22] H. B. Lawson and M.-L. Michelsohn, Spin Geometry. No. 38 in Princeton Mathematical Series. Princeton University Press, 1990.

[23] R. Swan, Vector bundles and projective modules, Trans. Am. Math. Soc. 105 (1962) 264-277.

[24] B. P. Dolan, I. Huet, S. Murray, and D. O'Connor, Noncommutative vector bundles over fuzzy $\mathbb{C P}^{n}$ and their covariant derivatives, JHEP 07 (2007) 007, [hep-th/0611209].

[25] U. Carow-Watamura, H. Steinacker, and S. Watamura, Monopole bundles over fuzzy complex projective spaces, J. Geom. Phys. 54 (2005) 373-399, [hep-th/0404130].

[26] H. Grosse, C. Klimcik, and P. Prešnajder, Topologically nontrivial field configurations in noncommutative geometry, Commun. Math. Phys. 178 (1996) 507-526, [hep-th/9510083].

[27] M. B. Green, J. H. Schwarz, and E. Witten, Superstring Theory. Vol. 2: Loop Amplitudes, Anomalies and Phenomenology. Cambridge Monographs On Mathematical Physics. Cambridge University Press, 1987.

[28] B. P. Dolan and C. Nash, The standard model fermion spectrum from complex projective spaces, JHEP 10 (2002) 041, [hep-th/0207078].

[29] T. Kugo and P. K. Townsend, Supersymmetry and the division algebras, Nucl. Phys. B221 (1983) 357.

[30] M. Cahen, A. Franc, and S. Gutt, Spectrum of the Dirac operator on complex projective space $P_{2 q-1}(\mathbb{C})$, Lett. Math. Phys 18 (1989) 165.

[31] M. Cahen, A. Franc, and S. Gutt, Erratum to 'Spectrum of the Dirac operator on complex projective space $P_{2 q-1}(\mathbb{C})$ ', Lett. Math. Phys. 32 (1994) 365.

[32] C. Bär, Metrics with harmonic spinors, Geometric And Functional Analysis 6 (1996) 899.

[33] S. Seifarth and U. Semmelmann, The Spectrum of the Dirac operator on the odd dimensional complex projective space $C P^{(2 m-1)}, 1993$. pre-print no. SFB-288-95.

[34] C. N. Pope, Eigenfunctions and spin ${ }^{\mathrm{c}}$ structures in $C P^{2}$, Phys. Lett. B97 (1980) 417. 
[35] H. Grosse and A. Strohmaier, Noncommutative geometry and the regularization problem of 4 d quantum field theory, Lett. Math. Phys. 48 (1999) 163-179, [hep-th/9902138]. 